\title{
Article \\ The Fluidization Effect of a Bilayer Membrane on a Fatty Acid Vesicle by a Detergent
}

\author{
Shogo Taguchi *(D), Yuta Kimura, Yasuaki Tachibana, Takuji Yamamoto and Kouji Maeda \\ Department of Chemical Engineering and Materials Science, Graduate School of Engineering, \\ University of Hyogo, Kobe 651-2197, Japan; ek20z006@steng.u-hyogo.ac.jp (Y.K.); \\ eq17p051@steng.u-hyogo.ac.jp (Y.T.); tyamamot@eng.u-hyogo.ac.jp (T.Y.); maeda@eng.u-hyogo.ac.jp (K.M.) \\ * Correspondence: taguchis@eng.u-hyogo.ac.jp; Tel.: +81-(0)79-267-4851
}

check for updates

Citation: Taguchi, S.; Kimura, Y.;

Tachibana, Y.; Yamamoto, T.; Maeda, K. The Fluidization Effect of a Bilayer Membrane on a Fatty Acid Vesicle by a Detergent. Crystals 2021, 11, 1023. https://doi.org/10.3390/ cryst11091023

Academic Editor: Abel Moreno

Received: 30 July 2021

Accepted: 23 August 2021

Published: 25 August 2021

Publisher's Note: MDPI stays neutral with regard to jurisdictional claims in published maps and institutional affiliations.

Copyright: (c) 2021 by the authors. Licensee MDPI, Basel, Switzerland. This article is an open access article distributed under the terms and conditions of the Creative Commons Attribution (CC BY) license (https:// creativecommons.org/licenses/by/ $4.0 /)$.

\begin{abstract}
A bicelle, which is a bilayer molecular assembly, can be prepared by fluidizing a vesicle in the presence of a detergent. We investigated the effect of two different detergents, 3-[(3cholamidopropyl)dimethylammonio]-2-hydroxypropane sulfonate (CHAPSO) and Triton X-100 (TX), on the formation of a bicelle from a vesicle containing oleic acid (OA) and the detergent molecules. The fluidization effect of the detergent was evaluated using the membrane packing density, which we measured using the fluorescent probe method with Laurdan, in conjunction with transmission electron microscopy to examine the morphology of the prepared bilayer molecular assemblies. As a result, it was discovered that the OA/CHAPSO system could form a heterogeneous phase with the highest packing density, implying that CHAPSO was the better detergent for a bicelle preparation, whereas the OA/TX system formed a disordered phase with the lowest packing density.
\end{abstract}

Keywords: fatty acid; detergent; bicelle

\section{Introduction}

A bilayer membrane structured molecular assembly is expected to be used as functional membrane materials, for example, in drug carriers and cosmetic agents [1,2]. A bicelle, which consists of a long-chain lipid and a detergent, is an effective parent for functional membrane materials $[3,4]$ because its bilayer membrane structure continuously forms the morphology, such as multilamellar vesicles, toroidal pore vesicles, a disk-like micelle, or a thread-like micelle, depending on its concentration and its composition ratio $[5,6]$. The morphological property of a bicelle has also been used in the crystallization of membrane proteins $[7,8]$. The crystal structure of the membrane protein supported on the bicelle has been analyzed by nuclear magnetic resonance spectroscopy $[7,8]$. It should be noted that the composition must maintain the bilayer membrane structure to use a bicelle as the parent. By adjusting the $\mathrm{pH}$ and salt concentration, a fatty acid, which is a single unsaturated chain, is known to form a bilayer membrane structure [9]. A fatty acid decomposed from food forms an oblate bicelle with a coexisting bile salt in the human duodenum [10]. The thermodynamic properties of multi-dispersed oblate bicelles with sodium oleate and a bile salt or an analogous detergent have been reported [11]. Given their molecular assemblies, a fatty-acid-rich bicelle and a detergent-rich bicelle coexisted. The fatty-acid-rich bicelle had a bilayer membrane structure derived from fatty acids [12] and could be used as the parent. We have previously used a fluorescent probe, 6-dodecanoyl$N, N$-dimethyl-2-naphthylamine (Laurdan), to analyze the phase state of the fatty-acid-rich assembly [13]. The ordered and the disordered phase were found to coexist in the bilayer of oleic acid (OA), as a fatty acid, in a solution of 3-[(3-cholamidopropyl)dimethylammonio]2-hydroxypropane sulfonate (CHAPSO), as a detergent. CHAPSO is composed of a rigid steroid structure with a hydrophilic and a hydrophobic surface, similar to bile salts. Because the critical micelle concentration of CHAPSO ( $\mathrm{CMC}_{\mathrm{CHAPSO}}$ ) is $6-8 \mathrm{mM}$, this experiment was carried out at a concentration of $20 \mathrm{mM}$ higher than $\mathrm{CMC}_{\mathrm{CHAPSO}}$ for the OA bilayer 
and the CHAPSO molecule to interact well. Here, an OA vesicle was used as a source of a bilayer membrane [14]. We also discovered that the size of the OA vesicle decreased as the OA to CHAPSO mixing molar ratio $\left(\mathrm{X}_{\mathrm{OA}}=[\mathrm{OA}] /([\mathrm{OA}]+[\mathrm{CHAPSO}])\right)$ decreased [13]. The size reduction demonstrated that the CHAPSO molecule acted as a detergent fluidizing the OA bilayer membrane.

The fluidization of the bilayer membrane is required for the formation of a bicelle. Membrane fluidization techniques are commonly used in biological chemistry to extract membrane proteins. However, the mechanism of membrane fluidization is dependent on the type of detergents used. For example, it was reported that the hemolytic effect of 3-[(3-cholamidopropyl) dimethylammonio] propanesulfonate (CHAPS) and Triton X-100 (TX) on a natural erythrocyte membrane showed a difference between each membrane fluidization process [15]. The structure of CHAPS is similar to bile salts. The hydrophobic surface of CHAPS was expected to incorporate into the erythrocyte membrane, whereas the hydrophilic surface was exposed to an aqueous solution [15]. Incorporating CHAPS reduced the packing density of the acyl chains in the erythrocyte membrane while leaving the apparent packing density of its membrane surface almost unchanged [15]. Conversely, TX has a large polar moiety and has been used as a powerful detergent in that region. TX was expected to penetrate deeply into the erythrocyte membrane, resulting in membrane fluidization [15]. The results showed that the fluidization process and the resulting bicelle morphology should be taken into consideration.

This study reports the size of the OA/detergent bicelle and the packing density demonstrating the phase state of the OA bilayer membrane on its bicelle prepared at the molar ratio, $X_{\mathrm{OA}}(=[\mathrm{OA}] /([\mathrm{OA}]+[\mathrm{CHAPSO}$ or $\mathrm{TX}]))$, of 0.30 or 0.70 . They were evaluated to propose an effective combination as the parent bicelle. Here, the fatty acid, OA, is a source of the bilayer membrane. CHAPSO and TX were also employed as detergent models. The dynamic light scattering (DLS) and transmission electron microscopy (TEM) were used to determine the size. Deconvolution of the fluorescent spectrum with Laurdan as a probe was used to determine the packing density of the OA molecules in the bicelle. Then, we compared the packing density of the obtained bilayer membrane with that of several phospholipid vesicles with well-defined phase states [16,17].

\section{Materials and Methods}

\subsection{Preparation of $O A /$ Detergent Bicelles}

The OA vesicle $(\mathrm{pH} 8.5,[\mathrm{NaCl}]=50 \mathrm{mM})$ was first prepared [14]. The OA vesicle contained dehydrogenated OA molecules, i.e., oleates, with a molar ratio of OA to oleate of 20:80 [18]. As shown in Figure 1, an OA/CHAPSO bicelle of $20 \mathrm{mM}$ was prepared as a source of an OA bilayer membrane by mixing a $20 \mathrm{mM} \mathrm{CHAPSO}$ or TX stock solution with a $20 \mathrm{mM} O A$ vesicle solution with the desired $X_{\mathrm{OA}}$ of 0.30 and 0.70 .

\subsection{Evaluation of the Morphology of OA/Detergent Bicelles}

DLS apparatus (Zetasizer Nano-ZS, Malvern Panalytical Ltd., Osaka, Japan) was used to measure the size of each OA/detergent bicelle at $298 \mathrm{~K}$. A TEM image (HF-2000, Hitachi Ltd., Osaka, Japan) was used to determine the morphology of each OA/detergent bicelle.

\subsection{Evaluation of the Packing Density in OA/Detergent Bicelles}

Laurdan is used as a fluorescent probe that reflects the polarity of a bilayer membrane [4]. A spectrophotometer (FP-8300, JASCO Co., Osaka, Japan) was used to measure the fluorescent spectrum of $1 \mu \mathrm{M}$ Laurdan excited at $340 \mathrm{~nm}$ in a bicelle of $20 \mathrm{mM}$. The spectrum was then deconvoluted to determine the intensities of the three moments, corresponding to the packing density of the bilayer membrane. A Gaussian distribution was used to determine each moment. The center of each moment was at $440 \mathrm{~nm}$ (moment $i$ ), $490 \mathrm{~nm}$ (moment ii), and $530 \mathrm{~nm}$ (moment iii). It was anticipated that the moments $i$ and ii would have shown the hydrophobic and hydrophilic states in the molecular assembly, respectively [19]. Here, the moment iii meant more hydrophilic molecular assembly de- 
rived from bulk oleate and OA micelles. Thus, we calculated the packing density of the $\mathrm{OA} /$ detergent bicelle based on the area ratio of the moment $i$ to $i i, A_{\mathrm{i}} / A_{\mathrm{ii}}$.

$$
y=a_{0} \exp \left[-\frac{1}{2}\left(\frac{x-a_{1}}{a_{2}}\right)^{2}\right]
$$

where $x$ is the arbitrary wavelength $[\mathrm{nm}], y$ is the intensity associated with $x[-], a_{0}$ is the amplitude [-], $a_{1}$ is the moment's center position [nm], and $a_{2}$ is the width [nm].

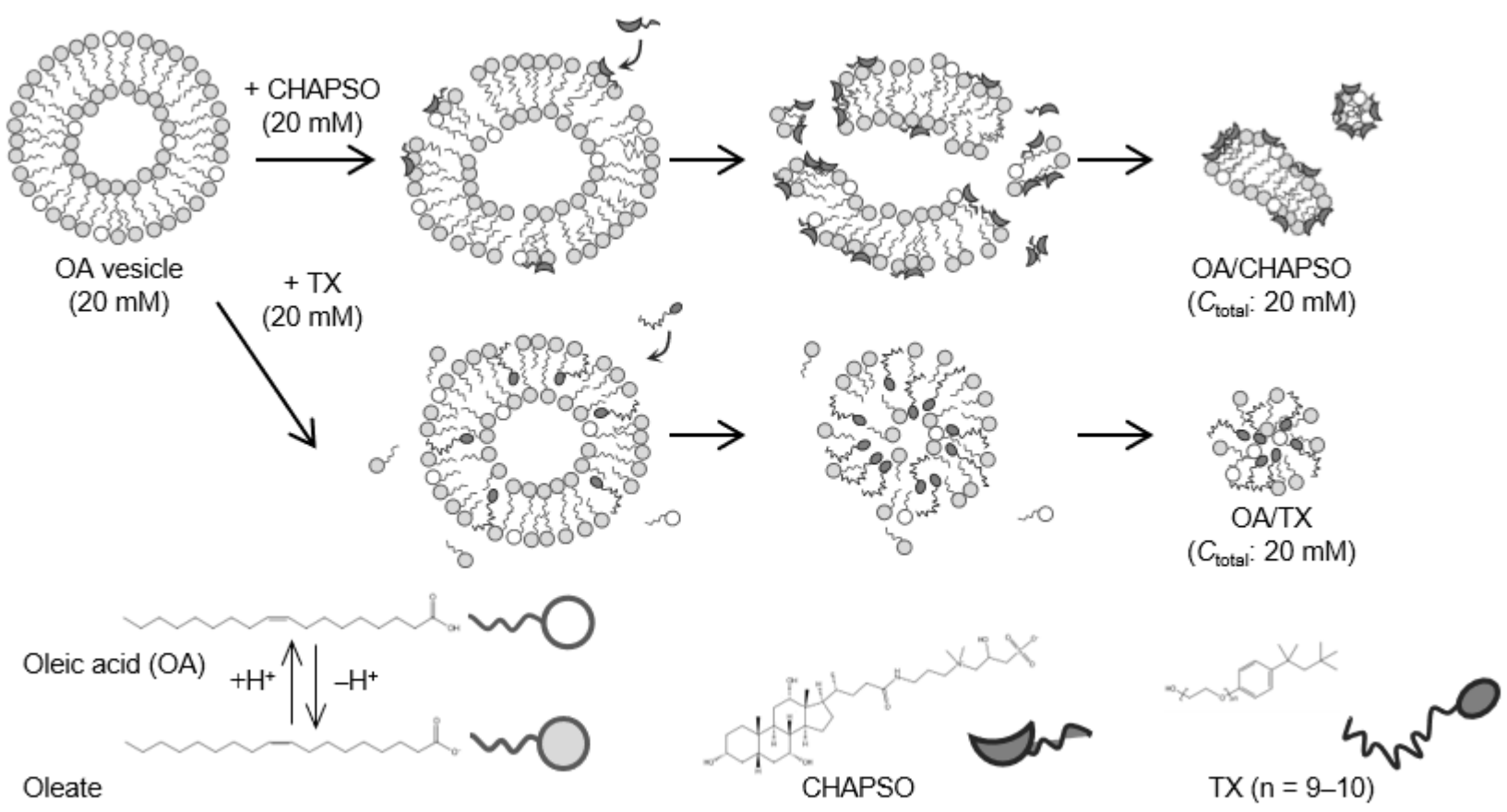

Figure 1. Outline of the OA/detergent (CHAPSO or TX) bicelle preparation.

\section{Results and Discussion}

\subsection{Morphology of OA/Detergent Bicelles}

DLS measurements were used to evaluate the formation of an OA/detergent bicelle. The size of an OA vesicle at $X_{\mathrm{OA}}=1.0$ reduced upon the addition of CHAPSO or TX solutions with decreasing $X_{\mathrm{OA}}$ to 0.30 and 0.70 (Figure 2). The size distributions with broad peaks also showed multi-dispersed bicelles. The results indicate the interaction between the detergent molecule and the OA vesicle, suggesting their potential for the formation of OA/detergent bicelles. When comparing the TEM images, it is apparent that the OA vesicle's spherical structure collapsed, resulting in non-spherical bicelles (Figure 3). However, the change in the morphology by fluidization was obviously different depending on the species of the employed detergent. Both detergent molecules can be electrostatically bound to the hydrophilic groups on the surface of the OA vesicle and the detergent changes the spherical morphology of the OA vesicle as a result of fluidizing the OA bilayer membrane $[15,20]$. The CHAPSO molecule on the OA bilayer membrane acted as a spacer and broke the OA vesicle's spherical morphology [15]. The hydrophobic chain of the OA molecule derived from the broken vesicle was then covered with another CHAPSO molecule to form an OA/CHAPSO bicelle that maintained the bilayer membrane structure [8]. On the other hand, despite the spherical appearance of the OA/TX bicelle, there was a swelling micelle in which the region of the bilayer membranes was expanded. TX molecules are thought to deeply penetrate the OA vesicle bilayer membrane [21], causing overall membrane fluidization [15]. The OA/TX bicelle is presumed to have shrunk by drawing the OA molecules from the OA bilayer membrane while penetrating the TX molecules. Although the different molecular assembly of $\mathrm{OA} /$ detergent can be formed by the addition of CHAPSO or TX solutions into the OA vesicle 
solution, the maintenance of the OA bilayer membrane structures requires further discussion in the next section.
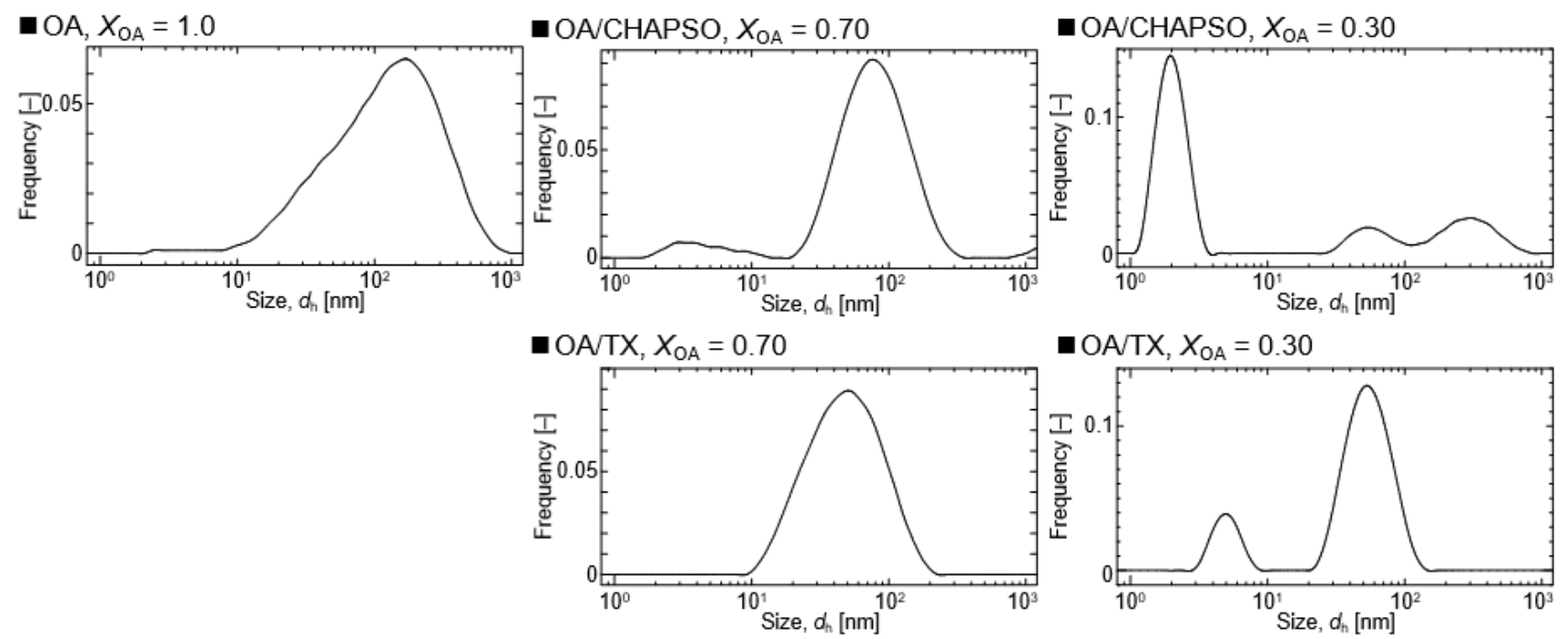

Figure 2. Size distributions of OA/detergent (CHAPSO or TX) bicelles at $20 \mathrm{mM}$ and at $298 \mathrm{~K}$.
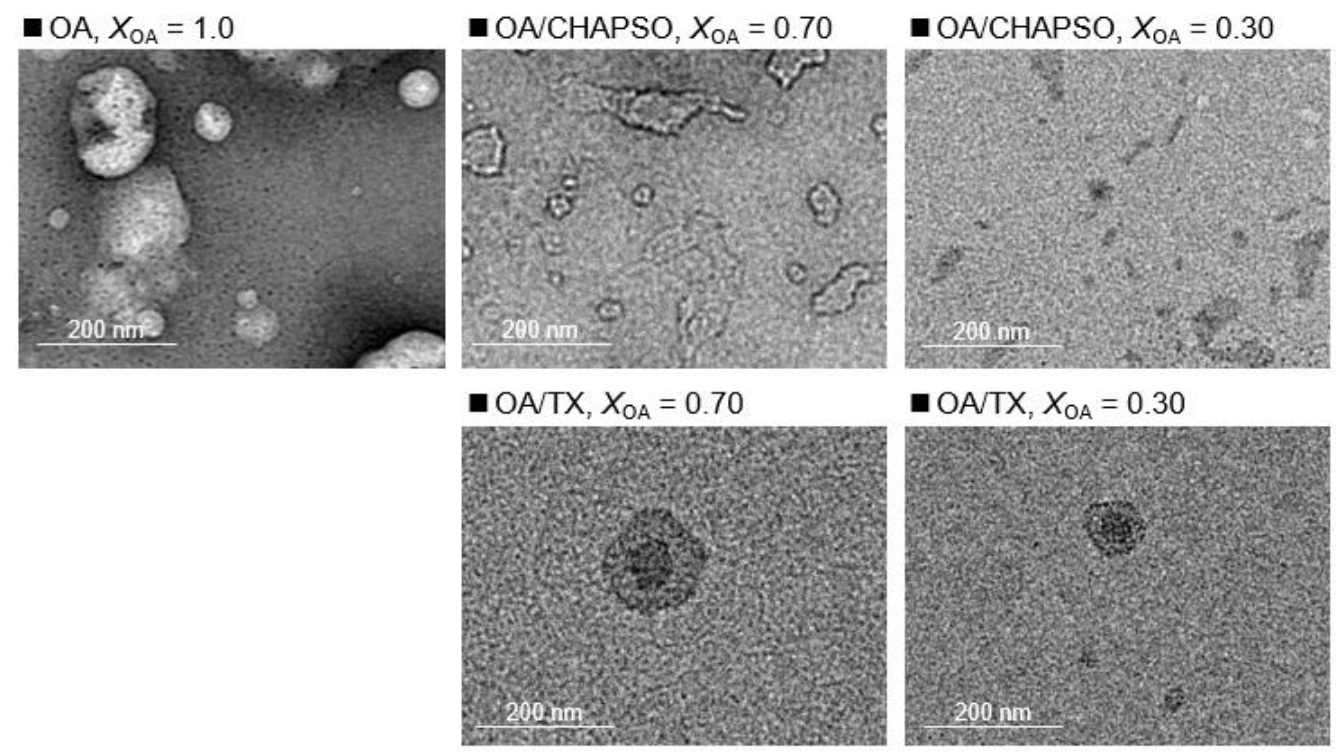

Figure 3. TEM images show a $20 \mathrm{mM}$ OA vesicle $\left(X_{\mathrm{OA}}=1.0\right)$, and $20 \mathrm{mM} \mathrm{OA} /$ detergent bicelles $\left(X_{\mathrm{OA}}=0.30\right.$ and 0.70$)$.

\subsection{Packing Density of OA/Detergent Bicelles}

To discuss the various phase states, we evaluated the packing density of the bilayer membrane structure. The packing density of the $\mathrm{OA}$ vesicle and the $\mathrm{OA} /$ detergent bicelle prepared in this study was compared to that of the previously revealed phospholipid vesicles $[16,17]$. The relationship between the packing density and the phase state in a bilayer membrane of phospholipid molecules can be explained as follows. At $298 \mathrm{~K}$, a densely packed bilayer membrane of a saturated phospholipid-e.g., 1,2-dipalmitoylsn-glycero-3-phosphorylcholine (DPPC)-keeps water molecules in an aqueous solution, and the inner DPPC membrane is hydrophobic. The DPPC membrane has an ordered phase. On the other hand, an unsaturated phospholipid-e.g., 1,2-dioleoyl-sn-glycero-3phosphocholine (DOPC) at $298 \mathrm{~K}$-forms a low packing bilayer membrane in an aqueous solution, and the inner DOPC membrane is hydrophilic because water molecules can easily penetrate the DOPC membrane. The DOPC membrane has a disordered phase. 
As a result, the packing density of a bilayer membrane is a function its phase state. In addition, depending on the ratio of DOPC to DPPC, a mixed vesicle of DOPC and DPPC (a DOPC/DPPC vesicle) has the ordered phase of DPPC and the disordered phase of DOPC-i.e., a heterogeneous phase [16,17]. The composition of the DOPC/DPPC ratio, $X_{\mathrm{DOPC}}(=[\mathrm{DOPC}] /([\mathrm{DOPC}]+[\mathrm{DPPC}]))$, can be used to prepare a model bilayer membrane in any phase state. The heterogeneous phase of the bilayer membrane on the DOPC/DPPC vesicle was reported to be in the $X_{\mathrm{DOPC}}$ range of $0.20-0.60[16,17]$.

As shown in Figure $4 a$, we measured the Laurdan spectra on DOPC/DPPC vesicles at various $X_{\mathrm{DOPC}}$ ratios. As a result of the deconvolution with Gaussian distributions, the spectra showed three moments. In the order shown from the left of Figure 4, moments $i$, $i$, and ii correspond to a hydrophobic bilayer membrane, a hydrophilic bilayer membrane, and a more hydrophilic assembly, such as a micelle [19]. Figure 4a shows that moment $i$ of the DPPC vesicle $\left(X_{\mathrm{DOPC}}=0\right)$ was stronger than the other moments, whereas moments ii and $i i$ increased as the $X_{\mathrm{DOPC}}$ ratio increased from 0 to 1.0. The DPPC vesicle has an ordered phase, and the unsaturated chain of the DOPC molecule also lowers the bilayer membrane packing and promotes the formation of the disordered phase [16-18]. Laurdan is frequently used to determine the phase state of phospholipid vesicles, using the peak intensity ratio between 440 and $490 \mathrm{~nm}[6,18]$. The relationship between $X_{\mathrm{DOPC}}$ and the estimated $A_{\mathrm{i}} / A_{\mathrm{ii}}$ ratio is shown in Figure $4 \mathrm{~b}$. We interpreted the phase state based on the ratio $A_{\mathrm{i}} / A_{\mathrm{ii}}$. Here, we ignored the influence of moment $i i i$, which could be attributed to micelle-like molecular assemblies, in calculating the $A_{\mathrm{i}} / A_{\mathrm{ii}}$ ratio from the deconvoluted spectrum of the detergent solution. We defined the threshold between the ordered phase and the heterogeneous phase, or the heterogeneous phase and the disordered phase, based on the $A_{\mathrm{i}} / A_{\mathrm{ii}}$ ratio of the DOPC/DPPC vesicles; (ordered phase) DPPC, (heterogeneous phase $)$ DOPC /DPPC $\left(X_{\mathrm{DOPC}}=0.20-0.60\right)$, (disordered phase $)$ DOPC, and DOPC $/ \mathrm{DPPC}$ $\left(X_{\mathrm{DOPC}}=0.80\right)$ at $298 \mathrm{~K}$.

(a)

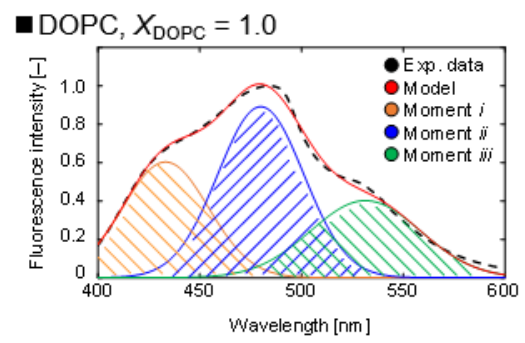

-DOPC/DPPC, $X_{\text {DOPC }}=0.60$
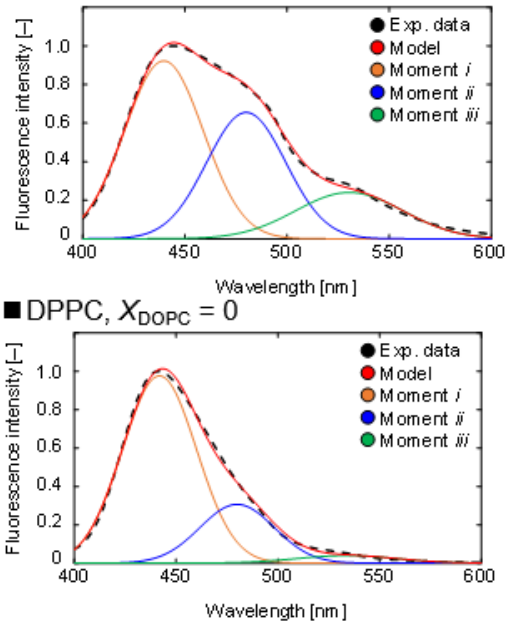

(b)

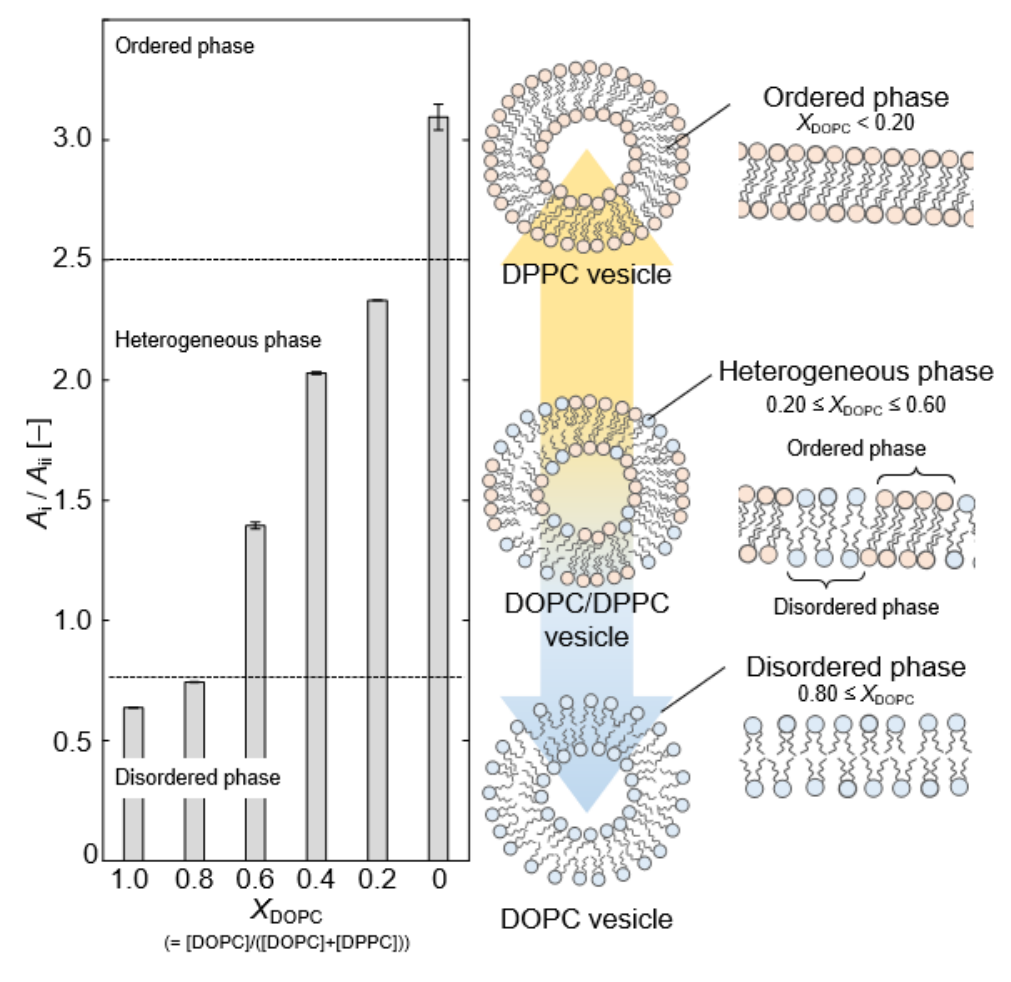

Figure 4. (a) Deconvolution results of Laurdan spectra by the measurement of a $2 \mathrm{mM}$ DOPC vesicle, a $2 \mathrm{mM}$ DOPC/DPPC vesicle $\left(X_{\mathrm{DOPC}}=0.60\right)$, and a $2 \mathrm{mM}$ DPPC vesicle at $298 \mathrm{~K}$. The experimental data (black) are fitted by the model (red), which consisted of moment $i$ (orange), moment $i$ (blue), and moment iii (green), respectively. (b) The relationship between $X_{\mathrm{DOPC}}$ and $A_{\mathrm{i}} / A_{\mathrm{ii}}$ ratio in the vesicles at $298 \mathrm{~K}$. The effect of the molecular structure of the phospholipids on the phase state of their vesicle is illustrated. 
Next, we evaluated the packing densities of the OA/detergent bicelles. The Gaussian distribution model was used to deconvolve each spectrum (Figure 5). At $X_{\mathrm{OA}}=1.0$, the OA vesicle's Laurdan spectrum showed three moments. From left to right, these moments denote a hydrophobic bilayer membrane, a hydrophilic bilayer membrane, and a micellelike assembly [19]. An OA vesicle solution contains OA molecules and oleate ions [18]. The coexistence of the OA molecule and the oleate ion results in forming a bilayer membrane structure of an OA vesicle, and the other oleate ion results in the formation of a micelle [18]. As a result, these three moments are included in the Laurdan spectra of the OA vesicle solution. The moments shown in Figure 5 indicate the presence of a micelle that is more hydrophilic than the OA vesicle in both CHAPSO and TX solutions at $X_{\mathrm{OA}}=0$. The areas were also different when comparing the moments of mixing CHAPSO and mixing TX at $X_{\mathrm{OA}}=0.30$ and 0.70 . When CHAPSO solution was added into the OA vesicle solution, the relative ratio of the moment $i$ to the moment $i i$ increased from that of the initial OA vesicle solution. In contrast, when TX solution was added into OA vesicle solution, the spectra were similar to that of the TX solution. The results imply that the phase state of the OA vesicle adsorbed to the detergents varied.
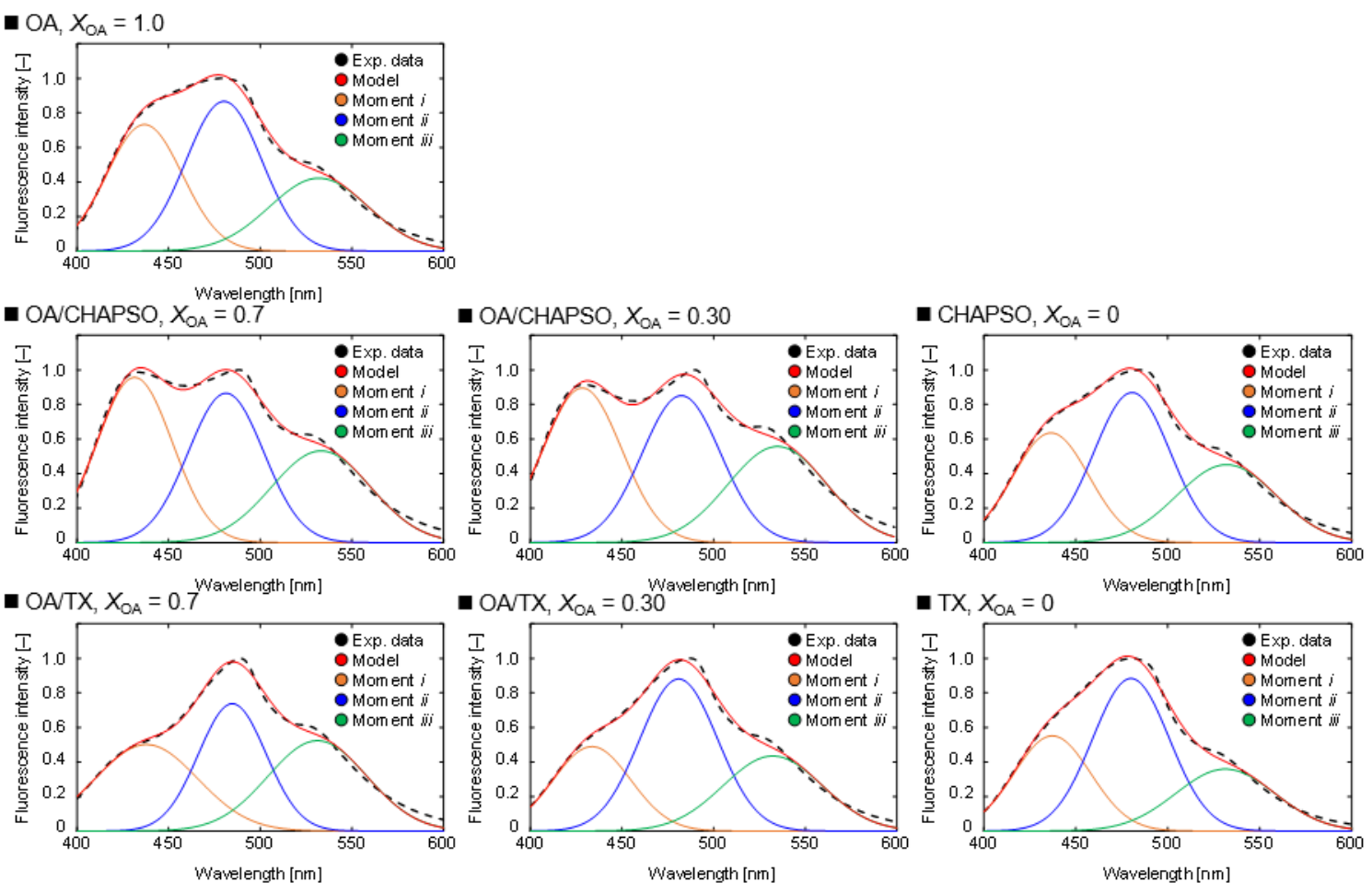

Figure 5. Deconvolution results of Laurdan spectra by the measurement of a $20 \mathrm{mM}$ OA vesicle $\left(X_{\mathrm{OA}}=1.0\right), 20 \mathrm{mM}$ $\mathrm{OA} /$ detergent bicelles $\left(X_{\mathrm{OA}}=0.30\right.$ and 0.70$)$ and detergent solutions $\left(X_{\mathrm{OA}}=0\right)$ at $298 \mathrm{~K}$. The experimental data (black) are fitted by the model (red), which consisted of moment $i$ (orange), moment $i$ (blue), and moment iii (green), respectively.

The OA vesicle's $A_{\mathrm{i}} / A_{\mathrm{ii}}$ ratio showed the heterogeneous phase (Figure 6). A portion of the OA molecule becomes an oleate, and there is an OA vesicle in which the oleate coexists [18]. When the CHAPSO molecules were mixed into the OA vesicles $\left(X_{\mathrm{OA}}=0.30\right.$ and 0.70 ), those packing densities were slightly improved (Figure 6). The result is expected to be due to the adsorption of the OA bilayer membrane by the CHAPSO molecule. As a spacer, the CHAPSO molecule electrostatically adsorbs on the hydrophilic group of the OA vesicle and can fluidize the OA vesicle's bilayer membrane structure [15]. In this case, the few OA molecules beneath the CHAPSO molecule are linked to a single CHAPSO molecule [22]. Given that the OA molecules beneath the CHAPSO molecule are difficult to fluidize [15,22], the OA bilayer membrane structure is thought to persist even after mixing a CHAPSO solution. Therefore, the $A_{\mathrm{i}} / A_{\mathrm{ii}}$ ratios at $\mathrm{X}_{\mathrm{OA}}=0.30$ and 
0.70 were more tightly packed-i.e., a more ordered phase state-than the OA vesicle. Adding a CHAPSO solution into an OA vesicle solution forms the OA/CHAPSO bicelle containing an OA bilayer membrane. However, the OA/TX bicelles with $\mathrm{X}_{\mathrm{OA}}=0.30$ and 0.70 showed disordered phases (Figure 6 ). Because TX molecules possessed a large polar moiety that can penetrate the OA bilayer, the entire OA molecule in the bilayer membrane was fluidized [15]. It can be considered that the OA/TX bicelle prepared at $X_{\mathrm{OA}}=0.70$ partially maintained the initial state of the OA vesicle. In contrast, the OA/TX bicelle prepared at $X_{\mathrm{OA}}=0.30$ suggested the formation of a mixed micelle and we could not conclude that the bilayer membrane structure was maintained. The bilayer membrane structure has been required to apply bicelles as a functional membrane material. Therefore, the OA/CHAPSO system, which can construct a non-spherical bicelle, is considered more suitable than the OA/TX system.
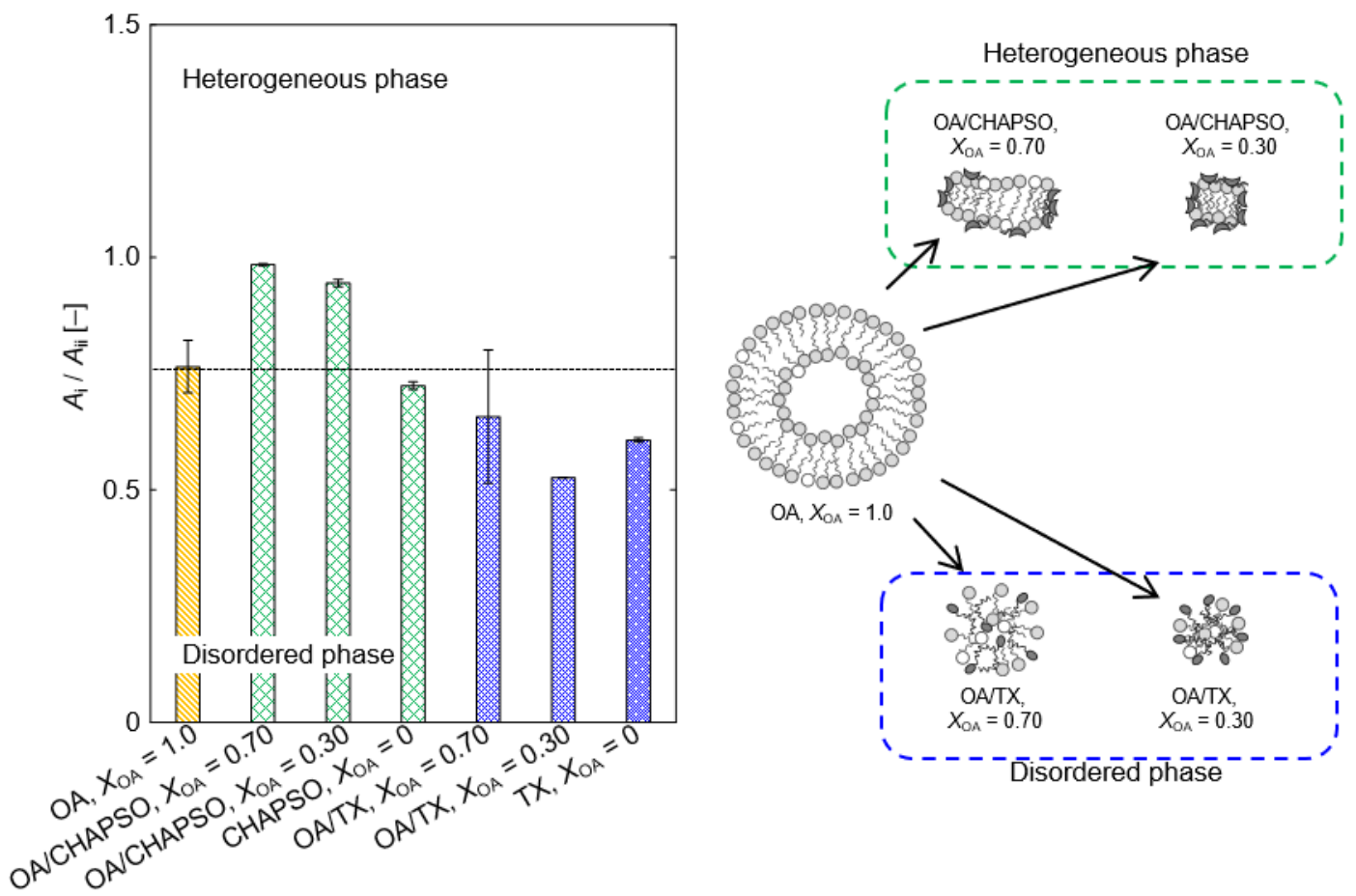

Figure 6. The relationship between $X_{\mathrm{OA}}$ and $A_{\mathrm{i}} / A_{\mathrm{ii}}$ ratio in OA/detergent bicelles at $298 \mathrm{~K}$. Each threshold of phase state was defined based on Figure 3.

\section{Conclusions}

The preparation conditions for the bilayer molecular assembly of a bicelle, using the fatty acid, OA, and the detergents CHAPSO or TX as a composition were investigated. The detergents fluidized the OA vesicles, and the sizes of the OA/detergent bicelles were determined by the molar ratios of the $\mathrm{OA}$ and the detergents. As a result of the phase state of the OA/detergent bicelles based on their packing densities, the fluidized OA bilayer membrane of the OA/CHAPSO system maintained a heterogeneous bilayer structure. However, mixed micelles were expected to be formed in the OA/TX system. It is suggested that these results were attributed to the detergents' fluidization processes, the fluidization process itself, and the resulting bicelle morphology. We believe that the OA/CHAPSO system is better suited for the application of functional membrane materials using the bilayer membrane structure of the bicelle.

Author Contributions: Conceptualization, S.T.; methodology, S.T.; formal analysis and investigation, S.T., Y.K. and Y.T.; writing—original draft preparation, S.T.; writing—review and editing, S.T., K.M. and T.Y.; supervision, S.T. and T.Y.; project administration, S.T.; funding acquisition, S.T. All authors have read and agreed to the published version of the manuscript. 
Funding: This research was funded by JSPS Grant-in-Aid for Early-Career Scientists (20K15129).

Acknowledgments: The authors thank Kaoru Mitsuoka (Research Center for Ultra-High Voltage Electron Microscopy, Osaka University) for TEM observation.

Conflicts of Interest: The authors declare no conflict of interest.

\section{References}

1. Pérez, B.; Bulsara, P.; Rawlings, A.V.; Wei, W.; Jensen, M.M.; Wang, Z.; Dickens, J.; Zhang, S.; Elliot, R.P.; Glasius, M.; et al. Ultralong fatty acyl derivatives as occlusive structure lipids for cosmetic applications: Synthesis and characterization. ACS Sustain. Chem. Eng. 2016, 4, 7137-7146. [CrossRef]

2. Prakash, A.; Zhu, H.; Jones, C.J.; Benoit, D.N.; Ellsworth, A.Z.; Bryant, E.L.; Colvin, V.L. Bilayers as phase transfer agents for nanocrystals prepared in nonpolar solvents. ACS Nano 2009, 3, 2139-2146. [CrossRef] [PubMed]

3. Sut, T.N.; Jackman, J.A.; Cho, N.-J. Understanding how membrane surface charge influences lipid bicelle adsorption onto oxide surfaces. Langmuir 2019, 35, 8436-8444. [CrossRef] [PubMed]

4. Taguchi, S.; Kang, B.-S.; Suga, K.; Okamoto, Y.; Jung, H.-S.; Umakoshi, H. A novel method of vesicle preparation by simple dilution of bicelle solution. Biochem. Eng. J. 2020, 162, 107725. [CrossRef]

5. Yamamoto, K.; Soong, R.; Ramamoorthy, A. Comprehensive analysis of lipid dynamics variation with lipid composition and hydration of bicelles using nuclear magnetic resonance (NMR) spectroscopy. Langmuir 2009, 25, 7010-7018. [CrossRef]

6. Taguchi, S.; Suga, K.; Hayashi, K.; Okamoto, Y.; Jung, H.-S.; Nakamura, H.; Umakoshi, H. Systematic characterization of DMPC/DHPC self-assemblies and their phase behaviors in aqueous solution. Coll. Interfaces 2018, 2, 73. [CrossRef]

7. Schmidt, T.; Situ, A.J.; Ulmer, T.S. Direct evaluation of protein-lipid contacts reveals protein membrane immersion and isotropic bicelle structure. J. Phys. Chem. Lett. 2016, 7, 4420-4426. [CrossRef]

8. Mineev, K.S.; Nadezhdin, K.D.; Goncharuk, S.A.; Arseniev, A.S. Characterization of small isotropic bicelles with various compositions. Langmuir 2016, 32, 6624-6637. [CrossRef]

9. Lu, H.; Shi, Q.; Huang, Z. pH-responsive anionic wormlike micelle based on sodium oleate induced by NaCl. J. Phys. Chem. B 2014, 118, 12511-12517. [CrossRef] [PubMed]

10. Hernell, O.; Staggers, J.E.; Carey, M.C. Physical-chemical behavior of dietary and biliary lipids during intestinal digestion and absorption. 2. Phase analysis and aggregation states of luminal lipids during duodenal fat digestion in healthy adult human beings. Biochemistry 1990, 29, 2041-2056. [CrossRef] [PubMed]

11. Hildebrand, A.; Garidel, P.; Neubert, R.; Blume, A. Thermodynamics of demicellization of mixed micelles composed of sodium oleate and bile salts. Langmuir 2004, 20, 320-328. [CrossRef] [PubMed]

12. Suys, E.J.A.; Warren, D.B.; Porter, C.J.H.; Benameur, H.; Pouton, C.W.; Chalmers, D.K. Computational models of the intestinal environment. 3. The impact of cholesterol content and pH on mixed micelle colloids. Mol. Pharm. 2017, 14, 3684-3697. [CrossRef]

13. Taguchi, S.; Kimura, Y.; Tachibana, Y.; Yamamoto, T.; Umakoshi, H. Preparation of bilayer molecular assembly from fatty acid and detergent. Kagaku Kogaku Ronbunshu 2021, 47, 51-56. [CrossRef]

14. Salentinig, S.; Sagalowicz, L.; Glatter, O. Self-assembled structures and pKa value of oleic acid in systems of biological relevance. Langmuir 2010, 26, 11670-11679. [CrossRef] [PubMed]

15. Rodi, P.M.; Bocco Gianello, M.D.; Corregido, M.C.; Gennaro, A.M. Comparative study of the interaction of CHAPS and Triton X-100 with the erythrocyte membrane. Biochim. Biophys. Acta-Biomembr. 2014, 1838, 859-866. [CrossRef]

16. Zhao, J.; Wu, J.; Heberle, F.A.; Mills, T.T.; Klawitter, P.; Huang, G.; Costanza, G.; Feigenson, G.W. Phase studies of model biomembranes: Complex behavior of DSPC/DOPC/cholesterol. Biochim. Biophys. Acta—Biomembr. 2007, 1768, 2764-2776. [CrossRef]

17. Uppamoochikkal, P.; Tristram-Nagle, S.; Nagle, J.F. Orientation of tie-lines in the phase diagram of DOPC/DPPC/cholesterol model biomembranes. Langmuir 2010, 26, 17363-17368. [CrossRef]

18. Suga, K.; Otsuka, Y.; Okamoto, Y.; Umakoshi, H. Gel-phase-like ordered membrane properties observed in dispersed oleic acid/1-oleoylglycerol self-assemblies: Systematic characterization using Raman spectroscopy and a Laurdan fluorescent probe. Langmuir 2018, 34, 2081-2088. [CrossRef]

19. Iwasaki, F.; Luginbühl, S.; Suga, K.; Walde, P.; Umakoshi, H. Fluorescent probe study of AOT vesicle membranes and their alteration upon addition of aniline or the aniline dimer p-aminodiphenylamine (PADPA). Langmuir 2017, 33, 1984-1994. [CrossRef]

20. Giacomelli, C.E.; Vermeer, A.W.P.; Norde, W. Micellization and Adsorption Characteristics of CHAPS. Langmuir 2000, 16, 4853-4858. [CrossRef]

21. Pantaler, E.; Kamp, D.; Haest, C.W.M. Acceleration of phospholipid flip-flop in the erythrocyte membrane by detergents differing in polar head group and alkyl chain length. Biochim. Biophys. Acta-Biomembr. 2000, 1509, 397-408. [CrossRef]

22. Schubert, R.; Beyer, K.; Wolburg, H.; Schmidt, K.-H. Structural changes in membranes of large unilamellar vesicles after binding of sodium cholate. Biochemistry 1986, 25, 5263-5269. [CrossRef] [PubMed] 